\title{
Advanced Technology Developments for Europa Lander and other In-Situ Ocean World Missions
}

\author{
Primary Author: Ray Crum \\ Institution: Jet Propulsion Laboratory(JPL), California Institute of Technology (CIT) \\ Phone: 626-390-9165, email: ray.crum@jpl.nasa.gov \\ Co-authors \\ Gary Bolotin/JPL-CIT \\ Erik Brandon JPL-CIT \\ Polly Estabrook/JPL-CIT \\ John Gallon/ JPL-CIT \\ Mellisa Heller/Sandia National Lab (SNL) \\ Lisa McCollum/ NASA Marshall Space Flight Center (MSFC) \\ Morgan Parker/ JPL-CIT \\ Miguel San Margin/ JPL-CIT \\ Lori Shiraishi/ JPL-CIT \\ Anthony Wong/ JPL-CIT
}

The research was carried out at the Jet Propulsion Laboratory, California Institute of Technology, under a contract with the National Aeronautics and Space Administration (80NM0018D0004).

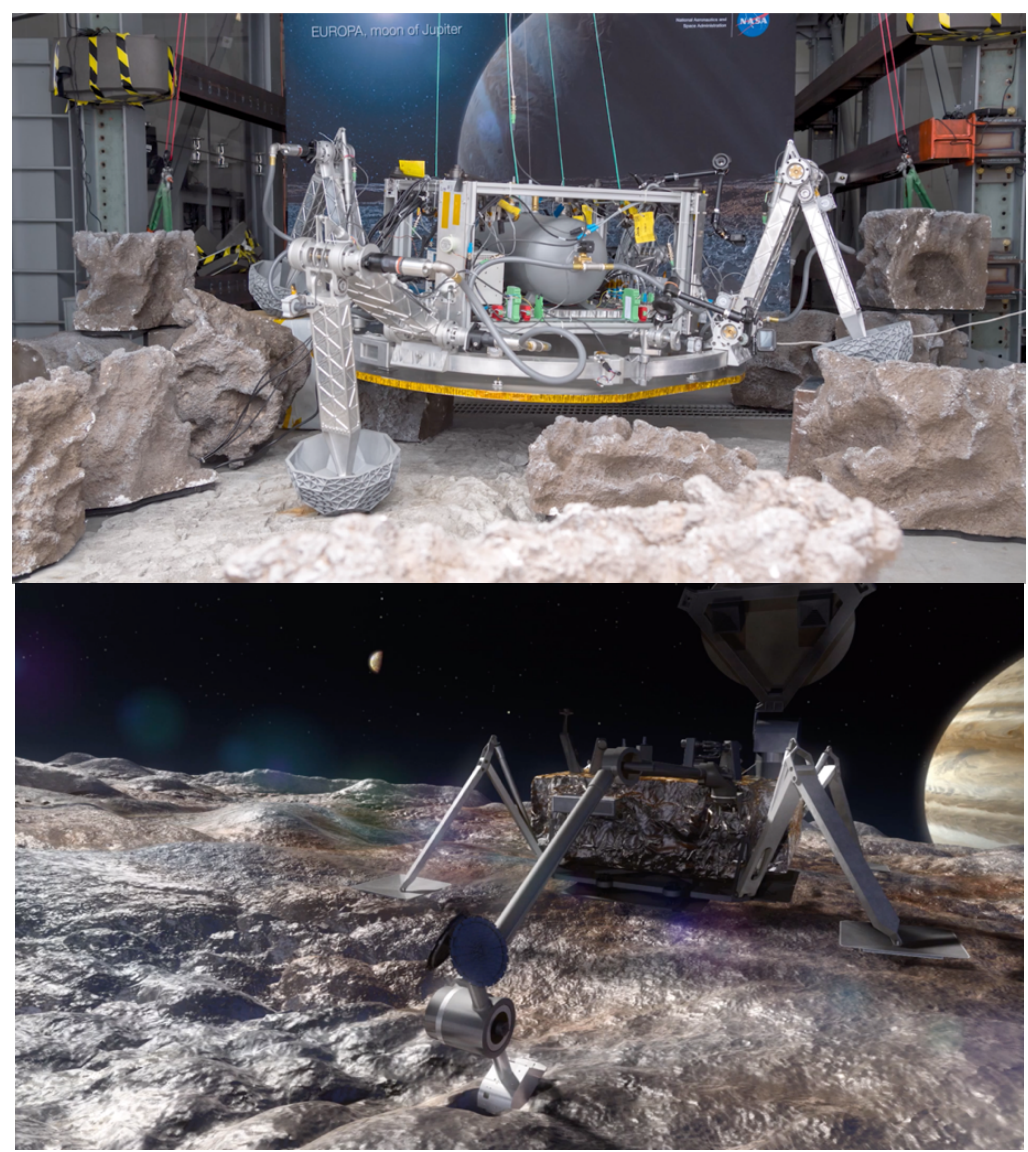

(C) 2020. California Institute of Technology. Government sponsorship acknowledged

Predecisional information, for planning and discussion only 


\section{Executive Summary}

The proposed Europa Lander mission concept targets civilization-scale changing science investigations on the surface of Europa. This mission concept passed its delta-Mission Concept Review (dMCR) in November 2018. Since that time, this pre-Phase A mission has continued maturing the technologies needed to implement the mission. In keeping with the tremendous potential science returns are several significant engineering developments. This paper details some of those developments and illustrates the work done by the Lander team to reduce the risk of these complex challenges particularly for De-orbit, Descent, \& Landing (DDL) and the surface phase of the mission.

Two complimentary white papers should be read in conjunction with this paper.

1) "Science of the Europa Lander Mission Concept", Hand, Kevin, et al.

2) "Development of Autonomous Actions to Enable the Next Decade of Ocean World Exploration”, Reeves, Glenn, et al.

In addition, detailed slides and a video describing the mission concept can be found here:

https://www.europa-insitu.caltech.edu/

The investments made in developments discussed in this white paper are for the: (1) deorbit stage, (2) landing engine, (3) landing lidar, (4) mechanical landing system, (5) contamination control, (6) landing system, (7) sampling system, (8) motor controllers, (9) batteries, (10) high gain antenna, and (11) planetary protection. The numbers refer to the section within this white paper. Figures 1 and 2 provide diagrams for these mission components. These investments by NASA will help enable a future Europa lander and other in-situ ocean world exploration missions.

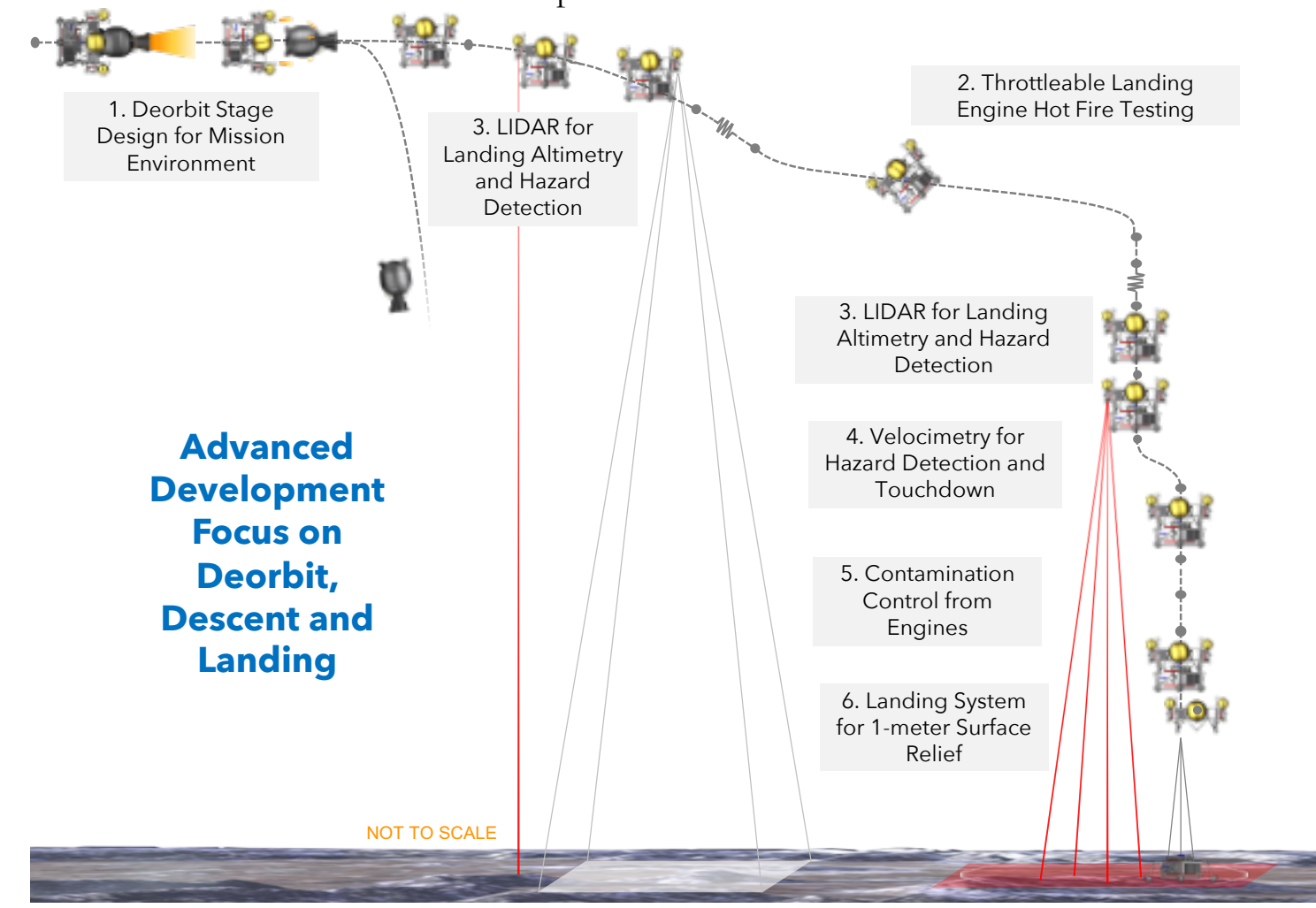

Figure 1. Advanced developments for the Europa Lander mission concept, as detailed in this paper. Shown above are developments 1through 6, all of which relate to the Deorbit, Descent \& Landing phase of the mission

(C) 2020. California Institute of Technology. Government sponsorship acknowledged

Predecisional information, for planning and discussion only 


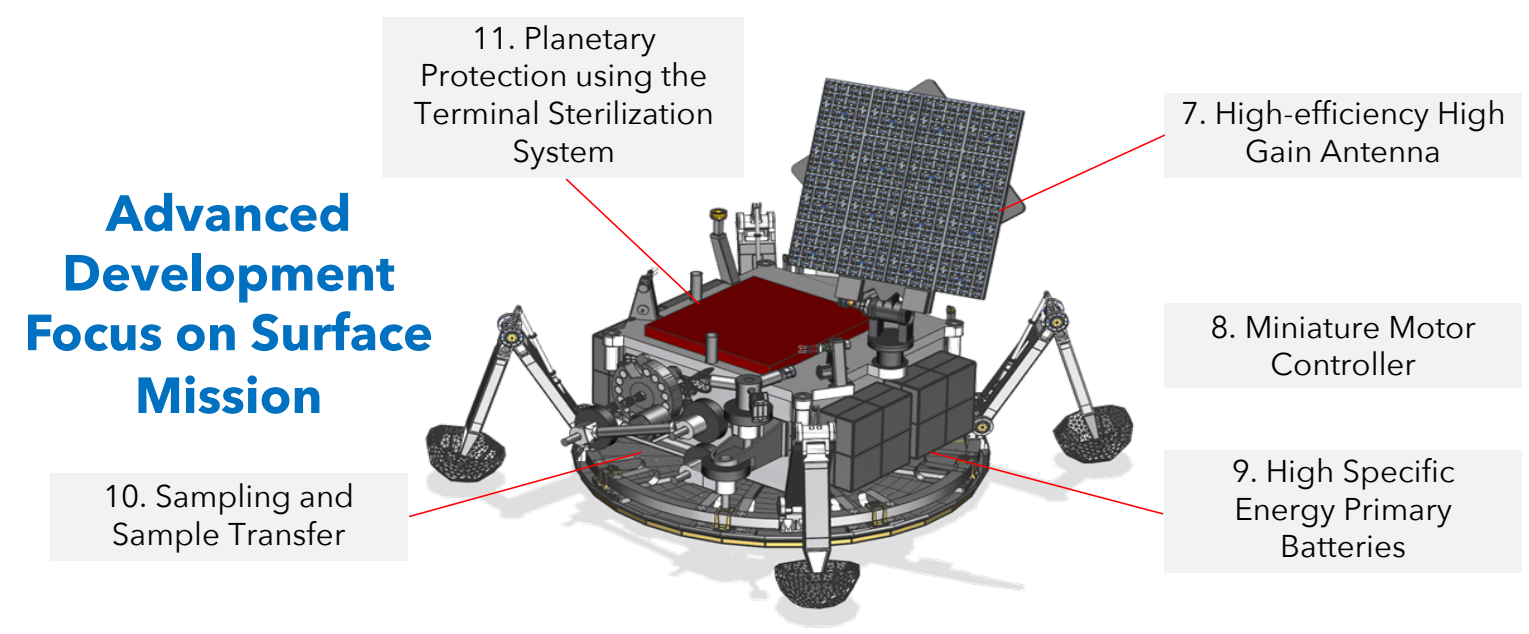

Figure 2. Advanced developments for the Europa Lander mission concept, as detailed in this paper. Shown above are developments 7 through 11, all of which relate to the surface phase of the mission

In addition to the advanced developments, this paper also describes the pre-projects top risks and mitigation strategies, including: 1) scope of new development, 2) radiation effect uncertainty, 3) compatibility with environment, 4) landing and sampling system tolerance to surface topography \& material properties, 5) sampling processing front end technology and 6) surface mission autonomy. The mission's major risk resides in two mission phases: DDL and surface science. For DDL, although we are leveraging existing technologies for the system design, the mass, environment, and rough surface require development. For the surface mission - the system design is driven by the mass, energy, environment, mission time, and unique surface properties.

The pre-project team ranked all of the mission risk mitigations and selected these 11 items for NASA's advanced development funding (Table 1). Work on these tasks began soon after the dMCR and will continue through at least 2022. A risk mitigation on mission autonomy is addressed in a separate white paper. When completed in 2022 all of these technologies will be at a TRL 6 .

\begin{tabular}{|c|c|c|c|}
\hline Item & Specific Risk Area & 18 Risk & 22 Risk \\
\hline De-Orbit Stage & Radiation, cold temperature, and long storage life & & \\
\hline Throttled Engine & Development of lower thrust engine & & \\
\hline Lidar & Environment, mass, dynamic range, and sample rate & & \\
\hline Velocimetry & Mass reduction & & \\
\hline Contamination & Contamination of samples by the propellant & & \\
\hline Landing System & Rough terrain with 1-m reliefs \& long-term stability & & \\
\hline High Gain Antenna & Limited communication time and distance & & \\
\hline Motor Control & Mass, volume and power reduction & & \\
\hline Batteries & Radiation and energy density & & \\
\hline Sampling System & Cryogenic material excavation, sampling, \& transfer & & \\
\hline Planetary Protection & Meeting $10^{-4}$ probability requirement & & \\
\hline
\end{tabular}

Table 1. Risk areas under development and mitigations as part of the effort described in this paper. Colors indicate the risk assessment in 2018 and project level by 2022. Some risks, such as the sampling system, will require instrument/payload selection before fully maturing to green.

(C) 2020. California Institute of Technology. Government sponsorship acknowledged

Predecisional information, for planning and discussion only 


\section{De-Orbit Stage Design for the Mission Environment}

Background and Rationale: The De-Orbit Stage (DOS) of the Europa Lander would use a solid rocket motor (SRM) to provide braking before the safe, accurate landing. Three unknown design factors for the DOS are the radiation, long-duration cold temperature, and planetary protection controls. We must characterize these environmental effects to validate or update the DOS design.

Development Approach and Results: We have engaged two suppliers, as well as analysis and testing at Marshall Space Flight Center (MSFC). MSFC developed a test plan to irradiate various SRM materials and perform complete post-irradiation evaluations of mechanical properties and ballistic performance. To date, these materials have included inert propellant, as well as propellant-linerinsulation (PLI) samples and live propellant. Radiation exposed samples showed an increase in hardening and a decrease in strain capability based on initial tests. The irradiated PLI bond line samples showed no significant degradation in bond strength. The results enabled the industry suppliers to select the best-performing formulation to continue testing and analysis. In the next phase, testing will focus on evaluating the effects of combined environments on propellant, the PLI bond line, and the ballistic performance of the DOS SRM. Radiation will be applied to samples along with cold and vacuum, which represent the transit and Jovian environments. The effects of these environments will be tested on a subset of inert SRM materials. At the end of the development, we will have concept design for the DOS from two suppliers with materials demonstrated to perform in the environments.

\section{Throttleable Landing Engine Hot Fire Testing}

Background and Rationale: A throttleable engine is necessary to provide the control authority needed for the descent profile demanded by the Europa Lander. The Mars Science Laboratory (MSL) $3300 \mathrm{~N}$ Mars Lander Engine (MLE) is too large to meet the requirements for the Europa mission. This development will modify the Throttle Valve of the MSL MLE for an $800 \mathrm{~N}$ engine.

Development Approach and Results: The first step of this development will modify the throttle valve assembly (TVA) by changing the cavitating venturi and pintle geometry to meet the smaller 800 $\mathrm{N}$ engine flow rate $(\sim 0.38 \mathrm{~kg} / \mathrm{s})$. Water flow testing will demonstrate that the modified TVA can achieve the required response time, throttle setting resolution, and flow rates, while maintaining cavitation over a full-throttle range 100\% to 1\%. The TVA will then be integrated with the engine and hot-fire tested to mimic the MSL MLE qualification test. These tests will demonstrate that the engine can achieve the quick response that GN\&C needs, and "deep throttle" from 100\% down to $\sim 15 \%$, with acceptable catalyst bed degradation (engine life verification). We are on contract with Moog; their TVA design is complete and the TVA material is ordered. The engine contract with Aerojet Rocketdyne has started.

\section{Dual-Mode Lidar for Landing Altimetry and Hazard Avoidance}

Background and Rationale: Even after the Europa Clipper mission, the lack of high-resolution (10's $\mathrm{cm}$ ), lander footprint compatible 3D terrain maps requires that the lander perform altimetry, real-time 3D mapping, hazard detection, and safe-site selection during DDL (a similar technique is used by Mars 2020). The short timeline for mapping and safe site detection over a $100 \mathrm{~m}$ by $100 \mathrm{~m}$ region at $5 \mathrm{~cm}$ ground sample distance requires a lidar that is capable of $4 \mathrm{Msamples} / \mathrm{sec}$. The lidar must be low mass and survive the radiation environment.

Development Approach and Results: We established a lidar development plan with two contractors followed by field tests to verify performance. The objectives of this plan are to: (1) identify optimal 
lidar modality; (2) develop designs and assess via component testing, analysis, and simulation; (3) identify critical technology enabling components of the lidar. Design, develop, and fabricate such components (e.g. large format focal plane arrays, ROICS and ASICS); (4) perform radiation testing on the critical components; and (5) demonstrate ranging and mapping performance over the DDL operational envelop of the flight-like lidars via field tests. The result of this development effort will be a lidar design that is space-qualifiable. We are currently in the second phase of the technology maturation with two vendors: Hexagon and MIT Lincoln Labs. After the delivery of the two brassboards, we will conduct field tests over Europan analogs for rough terrain and icy surfaces. This will use a helicopter-based platform for hazard mapping mimicking the descent profile at $500 \mathrm{~m}$ altitudes, and land vehicles for long-range $(5 \mathrm{~km}-8 \mathrm{~km})$ range acquisitions. The lidar-acquired digital terrain maps will then be compared against the truth map and the truth sensor suite on the test platform.

\section{Optical Velocimetry Eliminating the Need for a Landing Radar}

Background and Rationale: Prior landing systems have used onboard radar to obtain velocity measurements. To eliminate the mass of this radar, the lander would be the first interplanetary spacecraft to utilize a Terrain Relative Navigation camera and software to meet the $<0.1 \mathrm{~m} / \mathrm{s}$ velocity knowledge requirements. We will conduct a field test to prove out optical velocimetry.

Development Approach and Results: The team has developed an optical velocimetry software prototype, including image processing for feature tracking and state estimation, based on the Mars 2020 Lander Vision System. They conducted simulations for performance prediction and error analysis to quantify the effects of terrain relief, feature tracking errors, calibration errors, and IMU errors. For the Europa Lander field test, the team has developed a sensor payload, comprised of an inertial measurement unit, a 1-megapixel camera, laser rangefinder, and a GPS based inertial navigation system for ground truth. The payload will repeatedly execute terminal descents from $150 \mathrm{~m}$ altitude to soft touchdown, at vertical velocities of $5-10 \mathrm{~m} / \mathrm{s}$, over different terrain and under different lighting conditions. The data will be post-processed through the software prototype. Verifying that the test is consistent with the model predictions will allow the team to extrapolate to flight performance on Europa and inform algorithm refinements.

\section{Contamination Control from Engines}

Background and Rationale: Europa's surface would be impinged upon by the plumes of the Sky Crane engines that suspend the lander during its soft delivery to the surface. This plume impingement can contaminate or deform the surface. We need to understand the physical and chemical effects on the Europan surface of impinging combusted plume products, by-products, and unreacted precursor material. Creating an identification of the plume impingement products is essential to understanding the science returns from the lander mission.

Development Approach and Results: We will model engine plume expansion into vacuum during Sky Crane touchdown. We will use a methodology of one-way coupling between computational fluid dynamics and direct simulation Monte Carlo solutions that accurately represents both high-density, continuum plume flow at the exit of the engine nozzle and low-density, rarefied plume flow at the landing surface tens of meters away. This has been extended to incorporate realistic plume-plume and plume-vehicle interactions, with boundary conditions representative of those anticipated for the Lander. To validate the models, we will experimentally test analog monopropellant engine plumes with witness samples, and surface simulants to characterize the interaction properties. These properties will include position-dependent impingement velocities, droplet sizes, chemical

(C) 2020. California Institute of Technology. Government sponsorship acknowledged

Predecisional information, for planning and discussion only 
compositions, and chemical reactions. To date, we have developed simulations, obtained hydrazine thrusters, and have contracted for plume testing.

\section{Landing System for up to 1-meter Surface Relief}

Background and Rationale: The architecture utilizes the heritage MSL/M2020 Skycrane Landing System. It can support a touchdown of $\sim 0.5 \mathrm{~m} / \mathrm{s}$ vertical velocity. The four-legged landing gear provides adaptability to unknown $1 \mathrm{~m}$ surface reliefs with locking joints in the "hips" and "knees", which are commanded upon surface contact with the belly pan. Feet at the ends of the legs provides lateral grip and vertical reaction between the lander and the surface. Four bridles provide stability during initial terrain interactions to keep the vault level. Locking the leg joints results in a level and stable lander, transferring lander stability from the bridles to the Landing Gear. Once this transfer is complete and the Descent Stage (DS) flies away from the Lander.

Development Approach and Results: Landing system elements are being developed to demonstrate the performance of the landing system. This includes Foot Pad selection, Landing System prototypes, a Landing System Testbed, and a Landing Gear Testbed. The testing will show the impact and sensitivity of the Landing System elements to a simulated surface. The test data will be used to validate analytical models so that extreme cases can be evaluated with analysis. To date, a prototype Landing system has been designed and tested utilizing a full weight Lander simulator. The lander was successful at the full vertical landing speeds of $0.5 \mathrm{~m} / \mathrm{s}$. The overall Landing System performed as expected with no unexpected dynamics or fatal approach flaws. The landing legs demonstrated conformance with the reliefs and slopes requirements. It demonstrated the robustness of leg locking triggered by the Belly Pan's contact. It also demonstrated the post-landing Lander Deck angles of less than the 10-degree requirement. Multiple foot designs have been developed and some have been tested to simulate landing dynamics. The second prototype leg configuration has been designed and is in assembly. The second prototype Belly Pan is being designed and will enter fabrication soon.

\section{High-efficiency High Gain Antenna (HGA)}

Background and Rationale: The Europa Lander mission must support direct-to-earth communications and transmit 1.5 Gbit during surface operations. While most of the communications link can be supported with space qualified hardware, the requirements on the HGA drive an entirely different design. It must have high gain $(>36 \mathrm{dBi})$, high efficiency $(>75 \%)$, a low profile, be able to handle a 100-watt transmitter, and survive the high radiation and low temperature. The HGA chosen by Europa Lander is $32 \times 32$ patch array based on a newly patented High-Efficiency, Dual-Band Circular-Polarized Antenna for Harsh Environments.

Development Approach and Results: Using incrementally larger prototype antennas, the Lander team developed a plan to finalize the design for the $32 \times 32$ HGA and bring it to Technology Readiness Level 6. The test plan uses 8x8,16x16, and 32x32 element HGAs as test articles to verify requirements. An $8 \times 8$ array (with coax interface) verified performance within electrical and radiation requirements, and its mechanical design was validated at $-170 \mathrm{C}$. The $16 \times 16 \mathrm{HGA}$ gain was measured to be $31.3 \mathrm{dBi}$ at $8.425 \mathrm{GHz}$. To date, the 32x32 HGA has been designed, its parts fabricated, and the HGA assembled. Preliminary radiation patterns show excellent agreement with predicts. with gains of greater than $35.5 \mathrm{dBi}$ achieved. Once assembly is complete on the $32 \times 32 \mathrm{HGA}$, the antenna's electrical performance will be measured, and it will be tested in relevant mechanical environments. including pattern and gain measurements at $-170 \mathrm{C}$. Another $8 \mathrm{x} 8$ subarray prototype will be used to verify radiation susceptibility and dielectrics performance in the antenna.

(C) 2020. California Institute of Technology. Government sponsorship acknowledged

Predecisional information, for planning and discussion only 


\section{Miniature Motor Controller}

Background and Rationale: To allow more resources for lander instruments we are reducing the volume, mass, and power of its motor control. To achieve this reduction, we are developing modular standardized Multi-Chip Modules, utilizing advanced substrate and System-in-Package technologies that can be configured into a compact topology.

Development Approach and Results: The motor controller consists of a computer card, a power supply card, and enough motor control cards to control 12 motors. Many functions common to our previous motor controller designs implemented on Mars 2020 have been packaged into Multi-chip Modules. These functions include motor drive electronics, resolver electronics, power regulation, current sense, and telemetry electronics. These modules allow for considerable miniaturization of the electronics without losing functionality. The goal of this work is to design, build, and test a prototype motor control box that can be integrated into a field test demonstration in 2021. We have completed the requirements development and passed key milestone reviews. All modules have been designed and tested individually except the $10 \mathrm{~A}$ version of the motor driver module, which has not yet been tested. We have completed the fabrication of the first motor control card, and it is now being tested. The power conversion card is now in fabrication. The processor card is in design.

\section{High Specific Energy Primary Batteries}

Background and Rationale: Primary batteries have been baselined as the sole power source for the 20 day mission. The specific energy of cells of $>700 \mathrm{Wh} / \mathrm{kg}$ provides sufficient energy and stays within our battery mass envelope. The power modes of the lander would require only low discharge currents with the battery thermal design targeting operation over the range of 0 to $60^{\circ} \mathrm{C}$. Evaluation of various primary battery chemistries indicates that the $\mathrm{Li} / \mathrm{CFx}$ is the only chemistry that would meet or exceed the Lander requirements. Development is required since there is no flight heritage for Li/CFx.

Development Approach and Results: We established a test matrix for evaluating D-sized cells from several vendors. This testing included evaluations of cells exposed to twice the mission radiation dose. The most critical aspect of this testing is electrical performance testing over a range of operational temperatures and rates to establish the ability of this cell chemistry to meet different mission power profile scenarios. Total delivered energy is critical; we are testing to establish the mean and standard deviation of delivered energy and capacity of cells from different manufacturing lots. We will develop a statistical model that bounds the maximum and minimum energy delivered. We are characterizing the self-discharge and calendar life characteristics under long term storage conditions. Storage testing is currently being performed under real-time $\left(20^{\circ} \mathrm{C}\right)$ and accelerated $\left(30,40,60^{\circ} \mathrm{C}\right)$ conditions to extrapolate to 10 years of storage time. Waste heat from the battery discharge is used for lander heating; we are testing to determine the ratio of thermal to electrical power generated under different load conditions. Testing to date has indicated the Li/CFx chemistry meets or exceeds the load profiles and targeted mission duration for the Lander. A specific energy of $>700 \mathrm{Wh} / \mathrm{kg}$ at relevant rates has been demonstrated with minimal impact of $10 \mathrm{Mrad}$ radiation. Ongoing work is focused on longduration storage testing, evaluation of heat output vs. load, safety, abuse testing, and improved stateof-charge monitoring. Further cell design improvements are underway to improve specific energy. Based on the results so far, we expect to double the surface life from 20 to 40 days.

\section{Sampling and Sample Transfer}

(C) 2020. California Institute of Technology. Government sponsorship acknowledged

Predecisional information, for planning and discussion only 
Background and Rationale: We are exploring capabilities for autonomous excavation, collection, and transfer of the sample from an icy surface to the lander. The primary challenges associated with the Sampling Subsystem are (1) uncertain terrain topography, (2) uncertain material properties, (3) maintaining sample integrity throughout, (4) developing a system compatible with the harsh environment. Development work is underway to reduce these risks.

Development Approach and Results: A state-of-the-art cryogenic vacuum testbed (capabilities: $50 \mathrm{~K},<10 \mathrm{E}-6$ Torr) has been built, commissioned, and has demonstrated ice cutting in a cryo-vac environment. An extensive test campaign is underway to observe and characterize the behavior of cryogenic cuttings and test end-to-end sample integrity for all sampling processes (including mechanical and pneumatic solutions). In addition to the cryo-vac testbed, 6 ambient testbeds support sampling autonomy development, effects of system compliance, sampling robotic arm development, and initial tool investigations. Over 300 ambient tests have been completed to date, with significant long-term ambient test programs currently coming online. An initial cold actuator demonstration has occurred at $100 \mathrm{~K}$, vacuum. We are evaluating a range of tool options for excavation, collection, and testing those tools across a range of challenging surface simulants (varying both topography and material properties) to stress the capabilities of the sampling subsystem. Simulant compositions for cryogenic and ambient testing are being developed to identify key material properties that drive design and performance. At the task's end, we will demonstrate the capabilities of the sampling subsystem through a field test, create a Terrain Specification Document which outlines the range of simulant characteristics required for testing (mechanical properties, terrains, boundaries), and map sensitivities between surface properties, autonomy, and sampling hardware.

\section{Planetary Protection (PP) using a Terminal Sterilization System (TSS)}

Background and Rationale: PP regulations for icy moon landers require a sterility level on the hardware that would not be reached solely using ground bioburden reduction before launch. Radiation exposure (unique benefit of a Europa Lander) during the mission, would performs most of the bioburden reduction. At the mission's end, the TSS would sterilize the hardware protected from radiation in the vault. On command, energetic material (EM) would be ignited and raise the temperature of the components to achieve sterility within 14 seconds. Energic material development is required.

Development Approach and Results: The technical challenges to develop the TSS are (1) development of a new rapid heating EM formulation, (2) materials tests to the relevant environments, (3) development and validation of a model for the thermal response of electronics exposed to this $\mathrm{EM}$, and (4) testing of the packaged EM in a realistic configuration. The outcome of the current development work is a demonstration of a prototype that is at TRL-6. To date we have: (1) performed feasibility testing using available commercially available EM, (2) developed models to estimate the behavior and size of the system, (3) performed a material screening and down-selected the EM for the baseline design, (4) performed initial safety and sensitivity analysis on top EM, (5) designed and procured hardware for tests to verify the EM function and provide model validation data, and (6) developed a CFD model to predict the thermal response of sterilized materials. 